\title{
Energy-Efficient and Delay-Aware MAC Protocol in Wireless Sensor Networks for Oil and Gas Pipeline Monitoring
}

\author{
Huaping Yu, Mei Guo \\ College of Computer Science, Yangtze University, Jingzhou, 434023, China
}

Keywords: oil and gas pipeline, MAC protocol, energy efficient, delay aware, adaption.

\begin{abstract}
A flexible and energy efficient media access control (MAC) protocol is the fundament of wireless sensor networks for oil and gas pipeline systems. This paper firstly analyses the typical MAC protocols, three layers architecture of WSNs, and then proposes energy efficient and delay aware MAC (EEDA-MAC) protocol for oil and gas pipeline monitoring. Secondly, two frame structures of EEDA-MAC protocol for long line structure are introduced, and some improvement strategies for EEDA-MAC protocol are proposed and discussed such as energy consumption balance strategies, preambles mechanisms, adaptive and asynchronous competition mechanisms, ladder scheduling mechanisms, which can effectively prolong the network life span and improve the network delay performance.
\end{abstract}

\section{Introduction}

Pipeline transportation is the most economical way to transport crude oil, natural gas and chemical products due to its lower costs, higher capacity and better consistency than alternative transporting methods such as railroad and highway [1]. In recent years, Wireless sensor networks (WSNs) are developing into main technical method to solve the problem of various kinds of information monitoring [2]. WSNs consist of a large number of energy-limited sensor nodes, which are deployed with random or deterministic way in the monitoring area to automatically sense, process, and transmit all kind of monitored data, without any human intervention. It is obvious that WSNs have many advantages to construct pipeline monitoring systems in the terms of the cost performance, flexible and control efficiency, which can effectively improve the level of management and economic and social benefits. And furthermore, WSNs can work unremittingly without manual intervention and work at the night and abominable weather.

Media access control (MAC) protocol is the basis of the communication in WSNs nodes, which controls the wireless channel usage and resource allocation of WSNs, and then MAC protocol affects the energy efficient and delay performance of the network. Therefore, designing a kind of energy efficient and delay aware of MAC protocol to save node energy, prolong the network lifetime and meet delay performance is of great significance.

The paper is organized as follows. In the next section, we reviews related works. In Section 3, requirement analysis and related network models are given. Section 4 presents some strategies and the energy-efficient and delay-aware MAC protocol. Finally, the paper is concluded in section 5. etc.

\section{Related works}

\section{Energy consumption of sensor nodes.}

In general, the energy consumption of the sensor node is mainly from the wireless communication module (as shown in figure 1), and the communication module needs more electricity than sense and process modules. For example, the energy of transmitting 1 bit data is enough to perform 3000 computation instructions [3]. Furthermore, MAC protocol is one of the main factors of energy consumption of sensor nodes. 


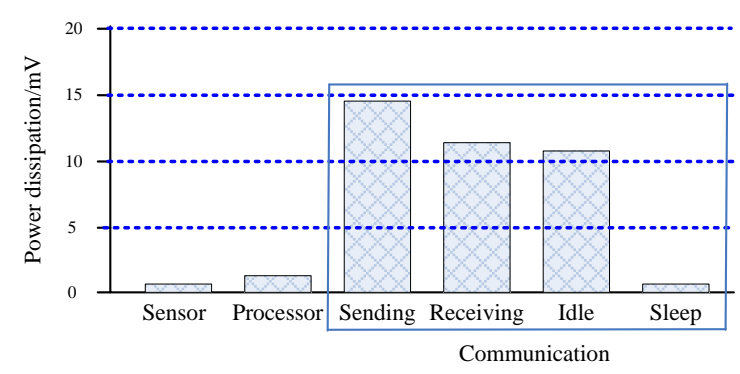

Fig.1: Energy consumption of sensor nodes distribution

In WSNs, the MAC protocol determines the energy consumption of sensor nodes, latency, and other performance indicators, which are the important indexes for designing MAC protocol. Energy waste of sensor node are mainly concentrated in the idle listening, message collision, overhead and control message overhead etc., and the idle listening is the dominant factor of energy consumption in MAC protocol. When designing a MAC protocol of WSNs, minimizing the idle listening of sensor node is very important on the basis of ensuring the time delay performance of the WSNs.

\section{Three types of MAC protocols.}

The MAC protocols are mainly classified into channel competition protocol, channel scheduling protocol and hybrid MAC protocol based on wireless channel access strategies (table 1). Channel competition protocols can be divided into asynchronous competition and synchronous competition. The typical MAC protocols includes: S-MAC [4], T-MAC [5], X-MAC [6] and B-MAC [7], which establish active/sleep state on demand, and their main problems are unnecessary energy consumption by idle listening and overhearing. Channel scheduling protocols are based on TDMA technology, and the sensor nodes transmit or receive packets in assigned time slots. The typical MAC protocols includes: L-MAC [8] and D-MAC [9], which access channel in turn according to the slots distribution, and have the characteristic of non-collision, delay-limit, flow stability and high load. Hybrid MAC is a new research direction of the MAC protocols with competition and fixed time slots allocation, which have the advantages of flexible and extensible channel allocation, low energy consumption and high load distribution. Crank-Shaft [10] and Z-MAC [11] protocols are the typical representative.

Table 1: Three types of MAC protocols

\begin{tabular}{ll}
\hline Protocol & \multicolumn{1}{c}{ Characteristic } \\
\hline S-MAC & Periodic listening/sleep \\
T-MAC & According to node traffic, adaptive adjust duty cycle \\
B-MAC & Low-power listening; Leading sequence length is not less than listen interval \\
X-MAC & $\begin{array}{l}\text { Flash frequency leading sequence; Listen early response effectively reduced the leading } \\
\text { sequence length }\end{array}$ \\
L-MAC & Node pre-allocated fixed time slot, reduced the online conflict. \\
D-MAC & Along the direction of data transmission, sensor nodes are waked up to work in turn \\
Crank-Shaft & $\begin{array}{l}\text { Node pre-allocated fixed time slot to minimize competition; Leading sequence initiate } \\
\text { Z-MAC }\end{array}$ \\
\hline
\end{tabular}

Oil and gas pipelines are typical long linear structure. There are some special MAC protocols of WSNs for linear structure monitoring such as SX-MAC [12], SLD-MAC [13]. SX-MAC protocol introduced synchronization and estimating waking up time based on X-MAC, and it can reduce the length of preambles and save energy to the maximum. In SLD-MAC protocol, when a source node transmits data to the gathering node, proper relay sensor nodes are selected for forwarding data according to the energy consumption factor of individual sensor node and residual energy balance factor. In this paper, we propose an energy-efficient and delay-aware MAC protocol (EEDA-MAC) of linear WSNs to save node energy, prolong the network lifetime and meet delay performance. 


\section{Network model}

\section{Requirement analysis.}

Oil and gas pipelines are made from steel or plastic tubes with inner diameter typically from 100 to $1,500 \mathrm{~mm}$. The oil is kept in motion by pump stations along the pipeline, and it usually flows at speed of about 1 to 6 meters per second. The gas is pressurized by compressor stations [1].

From the point of view of data collection requirements, data collection methods of pipeline state monitoring data can be divided into periodic data collection, event-driven data collection and query data collection. Periodic data collection is refers that the sensor node periodically sent the data to users according to the set sampling period (sending at a lower frequency, such as one hours, ten hours, so far as to one day); Event-driven data collection is refers that the sensor nodes continuously monitor sensing field (sampling at a higher sampling frequency, such as one second, one minute and so on), when the sensed data exceeds the certain thresholds, the related data will be transmitted to users and report the occurrence of relevant event immediately; Query data collection is refers that the sensor nodes send the monitored data to users according to the user's query command. From the point of priority of oil and gas pipeline state information, monitoring data packets may be classified into non-urgency data packets (e.g. soil parameters) and urgency data packet (e.g. oil and gas leak, soil parameters or quantity of flow and pressure state changes sharp). It is obvious that the nonurgency data packets are collected on periodic data collection modes, and the urgency data packets are collected on event-driven data collection and query data collection modes. Furthermore, when users need to get the information of specific sensing object, we can use the query data collection mode.

From the point of long linear-distributed sensor nodes, long linear structure leads the energy consumption of sensor nodes near the sinks to be consumed quickly and then soon to "death". The "death" of sensor nodes is fatal for the whole network because the data of other sensor nodes can't be sent to the sinks by means of the relay way. Therefore, the MAC protocol needs to consider the energy funnel problem.

Therefore, Influence factors in designing MAC protocol for oil and gas pipeline monitoring include traffic adaptive, delay performance and the energy funnel problem.

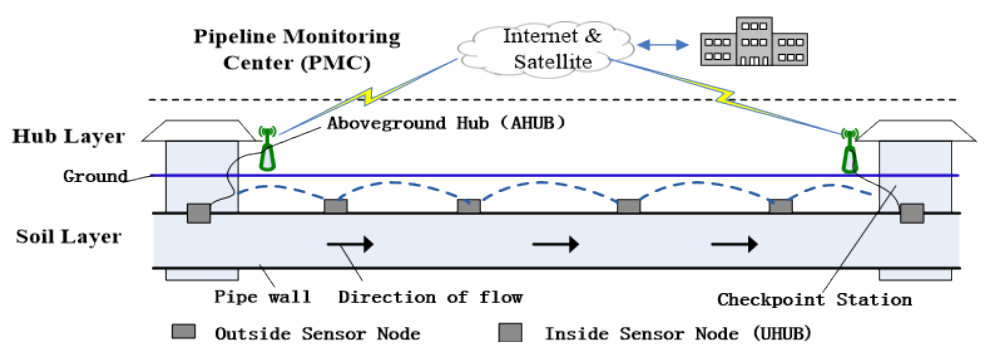

Fig.2: Network architecture

\section{Network architecture.}

Network architecture of WSNs is the foundation of MAC protocol, which includes three layers structure such as soil layer, hub layer and pipeline monitoring center. The three layers architecture is shown in figure 2.

\section{Energy-efficient and delay-aware MAC protocol discussion}

It is obvious that the long linear structure of oil and gas pipeline will lead to the problem of grievous non-uniformity energy consumption among the long linear-distributed sensor nodes and then subsequently reduce the lifetime of WSNs. In order to avoid unbalanced energy consumption in sensor nodes, we use the non-uniform line deployment strategy (figure 3) [14] and uniform deployment strategy, non-uniform partition strategy (figure 4) [15] to prolong the network life span. 


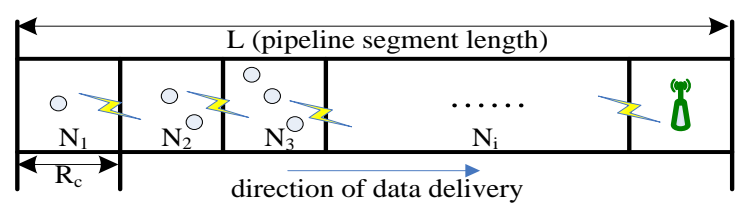

$\bigcirc$ Underground Sensor Node Intermediate Stations Nodes

Fig.3: Non-uniform deployment and scheduling strategy

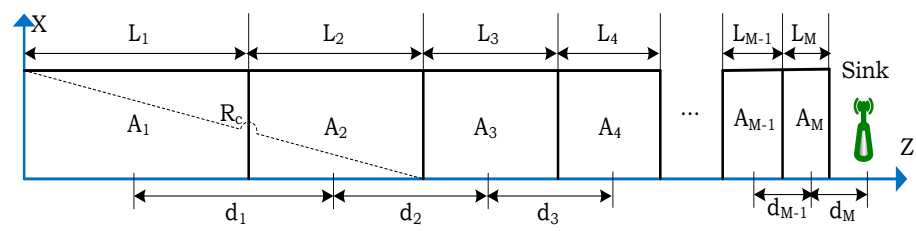

Fig.4: Uniform deployment and scheduling strategy

In figure 3 and figure 4, each partition elects cluster head nodes in turn, each cluster head is responsible for collecting data of intra-cluster node, and then transmit the data to gathering node through cluster heads with multi-hops ways. Therefore, the frame structure of EEDA-MAC protocol were designed into the pure CSMA mode in intra-cluster nodes (figure 5) and hybrid TDMA/CSMA mode in cluster heads (figure 6).

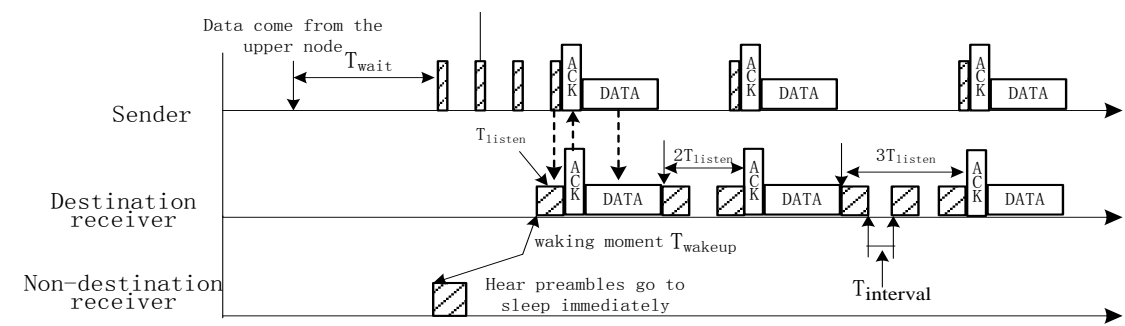

Fig.5: Frame structure for communication in intra-cluster nodes

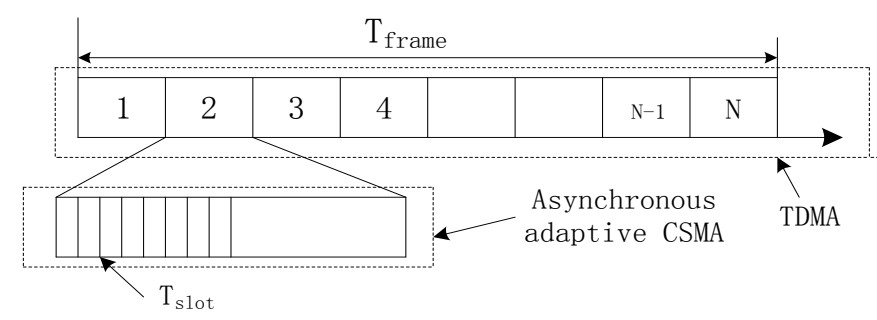

Fig.6: Frame structure for communication in cluster heads

\section{Preambles mechanism.}

Discontinuous preambles technology: In LPL mechanism [7], the destination receiver needs to listen the preambles until to the preambles end, which will cause the energy waste. Therefore, EEDA-MAC using discontinuous short preambles (figure 5) to save energy consumption, the source node listen the ACK from destination receivers between the slots of two adjacent short preambles.

Crosstalk avoiding technology: In order to avoiding the status of multiple nodes are listening the preambles simultaneously which will cause crosstalk interference and then lead to waste energy. In EEDA-MAC, each short preamble contains the address information of destination node. The nondestination node can sleep after listening to the preambles and avoiding the happening of crosstalk.

Data transmission volume feedback technology: The short preamble also contains the size information of the awaiting data transmission, which can be used to adjust the scheduling information of destination node.

\section{Adaptive asynchronous competition mechanism.}

Adaptive adjusting waiting time (Twait) technology in sender: In order to further reduce the length of the preambles, EEDA-MAC protocol sets up $\mathrm{T}_{\text {wait }}$, which is determined based on the waking 
moment of destination receiver ( $\left.T_{\text {wakeup }}\right)$. As shown in figure 5 , source node is in idle state in the time slot of $\mathrm{T}_{\text {wait }}$.

Adaptive adjusting listening interval $\left(T_{\text {interval }}\right)$ technology in receiver: If receiver has received data from sender in previous cycle, which shows that network load is heavy and $T_{\text {interval }}$ (figure 5) needs to be reduced. If the receiver does not receive data from sender in previous cycle, which shows that the network load is light and the $\mathrm{T}_{\text {interval }}$ needs to be increased.

Adaptive adjusting listening slot $\left(\mathrm{T}_{\text {listen }}\right)$ technology in receiver: The receiver continually increases $\mathrm{n}$ times listening after receiving the data from sending node. If receiver still can receive the data from sending node in the next greedy listening cycle, then the receiver continually and greedily listens until next listening cycle beginning, otherwise, the receiver turns to sleep until the next listen cycle beginning. Generally, the $\mathrm{n}$ is setted to 3 .

Destination node scheduling information feedback technology in ACK packet: The ACK can contain the active/sleep scheduling information of the receiver, which can be used to adjust the $\mathrm{T}_{\text {wait }}$ of preamble in sending node.

\section{Channel scheduling mechanism based on TDMA.}

For the characteristics of one-way routing, single-point convergence and fixing routing for oil and gas pipeline monitoring, the node cycle of EEDA-MAC protocol is divided into receiving slot, sending slot and sleep slot. In order to avoid idle listening further, EEDA-MAC protocol uses the ladder scheduling mechanism (figure 7). The data can be continuously transmitted to gather node along multiple hops path from source node to gather nodes, which can effectively reduce the transmission delay.

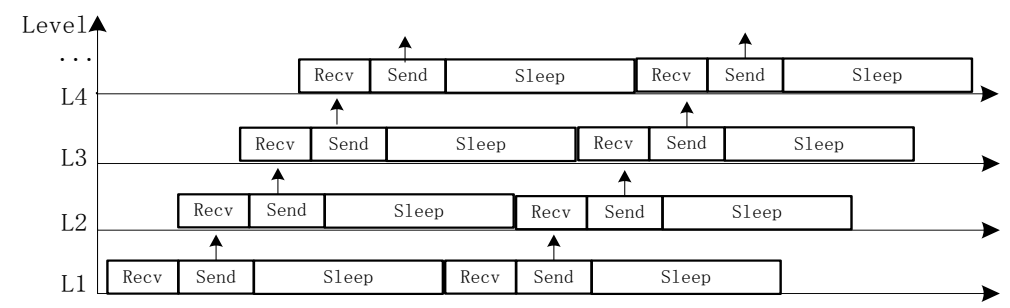

Fig.7: Ladder scheduling mechanism of EEDA-MAC for long line structure

\section{Conclusions}

In this work, we carefully analyzed typical MAC protocols and proposed three layers architecture of WSNs. In order to improve the energy efficient and delay performance of MAC protocol, we put forward EEDA-MAC protocol, which includes energy consumption balance strategies, preambles mechanism, adaptive asynchronous competition mechanism and ladder scheduling mechanism etc. In future, we plan to further investigate sensor node scheduling technologies of MAC protocol based on line structure to further enhance energy efficient and delay performance.

\section{Acknowledgements}

The authors are grateful to the anonymous referees for their valuable comments and suggestions. The research was supported by the PetroChina Innovation Foundation (Grant No.2013D-50060605).

\section{References}

[1]Y.Wikipedia.Pipelinetransport,(http://en.wikipedia.org/wiki/Oil_pipeline\#For_oil_or_natural_gas, 2014.12).

[2] I.F.Akyildiz and E.P.Stuntebeek. Wireless underground sensor networks: research challenges. Ad Hoc Networks, 4, pp. 669-686, 2006.

[3] H.P.Yu, C.X.Wu and M.Guo. Energy efficiency analysis for mobile wireless sensor network. 
Computer Engineering, 35(14), 127-129, 2009.

[4] Ye W., Heidemann J., Estrin D. Medium access control with coordinated, adaptive sleeping for wireless sensor networks. IEEE/ACM transactions on Networking, 12(3), pp. 493-506,2004.

[5] Dam T., Langendoen, K. An adaptive energy-efficient MAC protocol for wireless sensor networks. In Proceedings of the 1st International Conference on Embedded Networked Sensor Systems, pp. 171-180,2003.

[6] Buettner M., Yee G., Anderson E., et al. X-MAC: a short preamble MAC protocol for dutycycled wireless sensor networks. In Proceedings of the Fourth International Conference on Embedded Networked Sensor Systems, pp. 307-320,2006.

[7] Dutta P., Dawson-H S., Chen Y., et al. Design and evaluation of a versatile and efficient receiver-initiated link layer for low-power wireless. In Proceedings of the 8th ACM Conference on Embedded Networked Sensor Systems, 2010.

[8] Van Hoesel L.F.W., Havinga P.J.M. A light weight medium access protocol (LMAC) for wireless sensor networks: reducing preamble transmissions and transceiver stateswitches. In Proceedings of 1st International Workshop on Networked SensingSystems, 2004.

[9] Lu G., Krishnamachari B. An adaptive energy-efficient and low-latency MAC for data gathering in wireless sensor networks. In Proceedings of the 18th International Parallel and Distributed Processing Symposium, pp.224-231,2004.

[10] Halkes G.P., Langendoen K.G. Crankshaft: An Energy-efficient MAC protocol for dense wireless sensor networks. In Proceedings of the 4th European Conference on Wireless Sensor Networks, pp.228-244,2007.

[11] Rhee I., Warrier A. et al. Z-MAC: a hybrid MAC for wireless sensor networks. IEEE/ACM Transaction on Networking, 16(3), pp. 511-524, 2008.

[12] Zhu Bao-hui, Lin Jun-ru et al. An Improvement of MAC protocol for Transmission line monitoring in ldle period. Journal of Chinese Computer Systems, 35(5), pp. 1031-1036, 2014.

[13] Dai Guo yong et al. A MAC protocol for data gathering in linear wireless sensor network based on selective relay nodes. Chinsese journal of sensors and actuators, 27 (7), pp. 939-947, 2014.

[14] Yuan Hui-yong et al. Node deployment strategy in line wireless sensor networks. The journal of computer Engineering and Applications, 46 (7), pp.86-88, 2010.

[15] Huaping $\mathrm{Yu}$ et al. Deployment algorithms of wireless sensor networks for near-surface underground oil and gas pipeline monitoring. Sensors \& Transducers, 174 (7), pp.95-102, 2014. 